\title{
Identificación y control difuso de un variador-motor de inducción trifásico
}

\author{
Salatiel García-Nava, Julio C. Ramos-Fernández, Armando I. Martínez-Pérez, \\ Filiberto Muñoz-Palacios, Julio G. Duran-Candelaria \\ Universidad Politécnica de Pachuca, \\ Posgrado Maestría en Mecatrónica, Zempoala, \\ México \\ $\{$ sgn424,julio.duran.alpha\}@gmail.com, \\ $\{$ jramos, aimp, mupafi\}@upp.edu.mx
}

\begin{abstract}
Resumen. Un problema para diseñar estrategias de control a equipos mecatrónicos integrados con variadores de frecuencia y motores de inducción trifásicos, es la complejidad del modelado e identificación del variador electrónico de frecuencia, el motor de inducción trifásico y su interrelación. En el presente trabajo, se propone una metodología para identificar y controlar mediante un controlador PI con ganancias programables integrando la técnica difusa del tipo Takagi Sugeno, al conjunto variador de frecuencia y motor de inducción trifásico. Los resultados en simulación y en tiempo real del diseño del controlador propuesto para la velocidad del motor de inducción, indican que la metodología que se propone facilita el diseño del controlador, sin usar los modelos del variador de frecuencia y el motor de inducción que tienen representación no lineal. De los resultados del trabajo experimental realizado, el error cuadrático medio (RMSE) en la regulación para velocidades de referencia: 1100, 2050 y 3000 revoluciones por minuto (RPM) en estado estacionario corresponden a: 4.3, 10.76 y 13.09 respectivamente, adicionalmente se programó el algoritmo en un microcontrolador Cortex-M3 de 32bits en lenguaje C, que resulta en una implementación económica.
\end{abstract}

Palabras clave: PI con ganancias difusas programadas, motor trifásico, mecatrónica.

\section{Identification and Fuzzy Control of a Three-Phase Induction Motor with VFD}

\begin{abstract}
A trouble to design control strategies for integrated mechatronic equipment with frequency inverters and three-phase induction motors is the complexity of the modeling and identification of the electronic frequency inverter, the three-phase induction motor and its interrelation. In the present work, a methodology is proposed to identify and control by means of a PI controller with programmable gains integrating the fuzzy technique of the Takagi Sugeno type, to the frequency variator
\end{abstract}


set and the three-phase induction motor. The results in simulation and in real time of the design of the proposed controller for the speed of the induction motor, indicate that the proposed methodology allows the design of the controller, without using the models of the frequency inverter and the induction motor that have representation non-linear From the results of the experimental work carried out, the mean square error (RMSE) in the regulation for reference speeds: 1100, 2050 and 3000 revolutions per minute (RPM) in steady state correspond to: $4.3,10.76$ and 13.09 respectively, it was also programmed the algorithm in a 32-bit Cortex-M3 microcontroller in $\mathrm{C}$ language, which results in an economical implementation.

Keywords: fuzzy PI gain scheduling, three-phase motor, mechatronics.

\section{Introducción}

En el sector industrial es ampliamente conocido el uso de controladores industriales conocidos como Variador de Frecuencia (VF) para controlar torque y velocidad del Motor de Inducción Trifásicos (MIT), el diseño de controladores se lleva a cabo a razón de la experiencia de los ingenieros de automatizacion y mantenimiento que no se encuentra familiarizados en el área de control. Por otro lado para aplicar estrategias de control a estos equipos es necesario conocer el modelo matemático del conjunto Variador de Frecuencia-Motor de Inducción Trifásico (VF-MIT) que es expresado con ecuaciones diferenciales no lineales [5], por ello es necesario conocer los parámetros correspondientes del motor como del variador, esto implica realizar pruebas de: rotor bloqueado, sin carga y de corriente directa $[1,6]$. Dichas pruebas requieren de la manipulación física del motor, lo que no es viable de realizar para los operarios en las líneas de producción industrial, aquí es donde existe la necesidad de una metodología que permita al operario el diseño de controladores en sitio de una forma rápida y segura con técnicas de control inteligente.

Existen técnicas para el control de motores de induccion trifasicos; el control por medio de funciones de activación wavelet [3], controles difusos aplicados a motores DC [7]. Se han elaborado trabajos referentes al control de motores de inducción de 3 fases usando algoritmos genéticos implementados mediante una PC y microcontroladores de la familia MCS-51 donde el algoritmo genético es encargado de seleccionar las ganancias Kp y Ki [8], control por medio del control de ángulo de fase para una fuente de fase simple [9], métodos sintetizados basados en puntos de vista optimos de teoría de control [11], técnicas de control implementado en controladores por medio de IGBT basado en control de velocidad sin sensores con la técnica de control por vector $[4,10]$, control con lógica difusa usando métodos de campo orientado para el control de velocidad utilizando variables lingüísticas en lugar de numéricas [12], implemetación de técnicas difusas basadas en la técnica indirecta de orientación del campo del rotor [13], controles aplicados a drivers AC [5] que se aplican a través de sus modelos no lineales. 
Por lo anterior, el presente trabajo proporciona una metodología que facilita la realización de pruebas de identificación en sitio al conjunto VF-MIT, lo que hace incluir las dinámicas de carga a las que el motor este acoplado, esto para brindarle una herramienta que ayuda a desarrollar controladores para procesos industriales, obteniendo un procedimiento fácil, rápido y económico durante la implementación. La aplicación de esta metodología de identificación y control utiliza las mediciones correspondientes a una entrada de aplicada (voltaje de 0 a 10V) y salida (Velocidad Angular) del conjunto VF-MIT, necesarias para realizar una identificación y aproximación lineal de submodelos utilizados para la aplicación de técnicas difusas de tipo Takagi Sugeno (TS).

El orden del artículo se presenta de la siguiente forma; materiales y métodos utilizados para llevar a cabo este trabajo son descritos en la Sección 2. En la Sección 3, se hace la descripción de la metodología empleada, Las simulaciones llevadas a cabo así como los resultados experimentales obtenidos se describen en la Sección 4. Finalmente, las conclusiones y trabajos futuros son presentados en la Sección 5.

\section{Materiales y métodos}

En la Figura 1 se aprecia la plataforma experimental. Se acopla de forma mecánica (1) al eje del motor un encoder modelo LPD3806-600BM-G5-24C encargado de medir la velocidad angular del MIT, un VF modelo Power Flex 525 de la marca Allen-Bradley (2) con terminales eléctrica para el voltaje de control de 0 a 10V, encargado de regular la velocidad del MIT. También se observa la tarjeta electrónica para la adecuación de señales y el microcontrolador empleado (3), el MIT es de la marca $S I E M E N S$ de $2 \mathrm{HP}$ (4). y por último un interruptor de encendido(5).

El algoritmo de identificación y control se programo en un microcontrolador Cortex-M3 de 32bits en lenguaje C, las conexiones se observan en la Figura 2.

\subsection{Plantilla para funciones de membresía}

El primer enfoque supone que el experto puede proporcionar inicialmente las funciones de membresía y las reglas difusas. Los dominios de las variables antecedentes de las reglas difusas, pueden dividirse simplemente en un número específico de funciones de membresía configuradas equidistantes como se realiza en el presente trabajo. La base de reglas se puede establecer para cubrir todas las combinaciones de los términos de antecedentes. En la literatura, este enfoque se denomina modelado difuso basado en plantillas.

La tarea de identificación es, entonces, estimar los parámetros restantes en el modelo difuso a partir de las mediciones experimentales. Con respecto al tipo de modelo difuso presentado en este trabajo, estos parámetros incluyen las reglas en el modelo difuso TS, y la relación que define la regla basada en modelos difusos relacionales y lingüísticos como se muesta en [2]. 


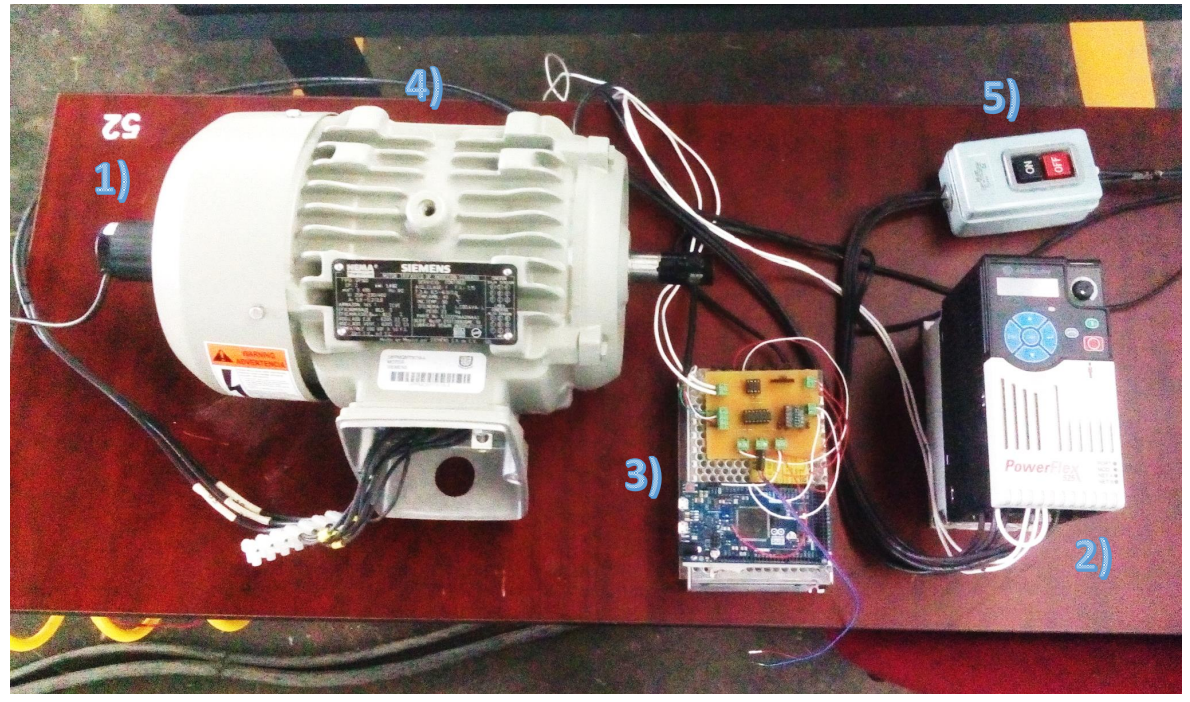

Fig. 1. Plataforma experimental utilizada para llevar a cabo el control de velocidad.

Se ha generado un conjunto de datos de entrada y salida a partir de su identificación. Conociendo que el sistema por observación de la respuesta a la entrada escalón, se puede aproximar con sub-modelos de primer orden, entonces la salida de cada región de operación identificada se puede modelar con la estructura de regla difusa mostrada en ecuación (1):

$$
\text { Si } y(k) \text { es } A_{i} \text { Entonces } y_{i}(k+1)=a_{i} y(k)+b_{i} u(k)
$$

donde:

$y_{i}(k+1)$ representa la aproximación por la i-ésima regla,

$a_{i}$ es el coeficiente de la entrada,

$b_{i}$ es el coeficiente del actuador,

$u(k)$ es la ley de control.

\section{Metodología de identificación y control}

La metodología que este trabajo propone a seguir se describe con el seguimiento de los siguientes pasos:

1.- Realizar pruebas del equipo mediante la aplicación de un algoritmo de identificación en lazo abierto al conjunto MIT-VF aplicando la cantidad de señales de excitación en función de las dinámicas de interés a modelar, en este trabajo se proponen 3 escalones de $3 \mathrm{~V}, 6 \mathrm{~V}$ y $9 \mathrm{~V}$ que de manera lingüística corresponden a baja, media y alta velocidad respectivamente, cada escalón con tiempo definido de tal forma que se pueda apreciar que la variable a 


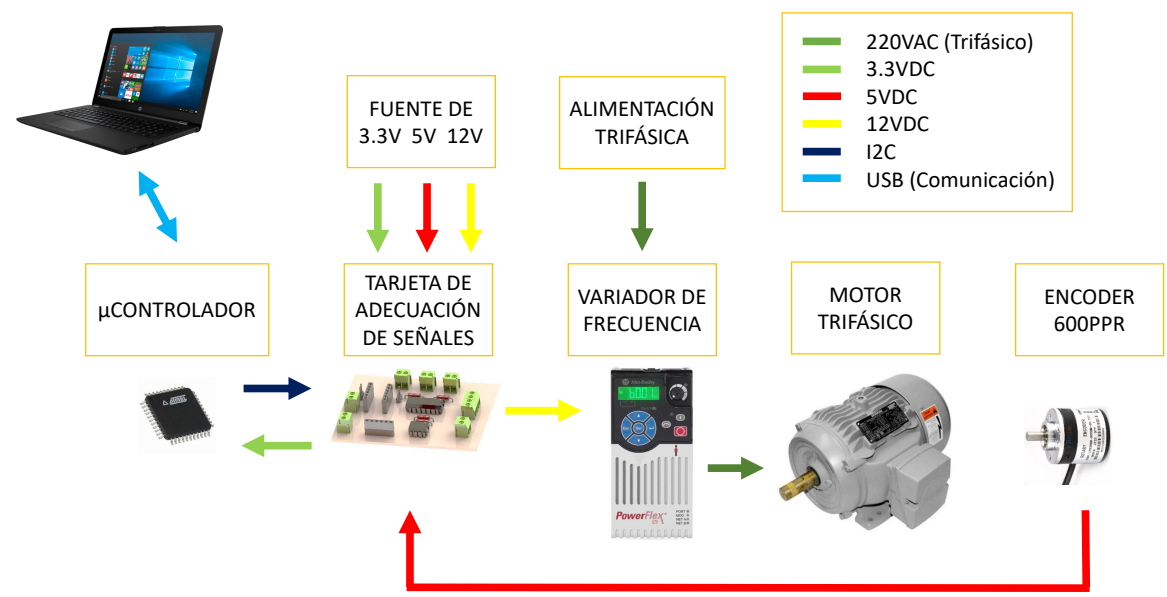

Fig. 2. Esquema de conexiones de la plataforma experimental para el control de velocidad del conjunto VF-MIT.

controlar sea estacionaria, el algoritmo de identificación permite obtener datos de entrada(voltaje aplicado) y salida medidos en el conjunto VF-MIT (velocidad angular) .

2.- Con las mediciones experimentales obtenidas de la respuesta a los diferentes escalones de excitación a la planta, mediante una supervisión gráfica se observa que las respuestas de cada escalón son cuasi-lineales así que se utiliza la herramienta de identificación de MATLAB; ident para obtener aproximaciones a sub-modelos de primer orden para cada sección, mostrados en las ecuaciones $(2,3,4)$ :

$$
\begin{aligned}
& \frac{R P M(s)}{V(s)}=\frac{730,9492}{s+1,9928}, \\
& \frac{R P M(s)}{V(s)}=\frac{1120,9664}{s+3,2978}, \\
& \frac{R P M(s)}{V(s)}=\frac{948,4271}{s+2,8418} .
\end{aligned}
$$

En la Figura 3, se observa la respuesta de los sub-modelos contra los datos de identificación obtenidos.

3.- Cada sub-modelo tiene una aproximación lineal, que se le sintoniza un controlador PI para este estudio de caso, sin limitar el uso de otro tipo de controlador, el diagrama a bloques muestra la implementación llevada a cabo en este trabajo que se observa en la Figura 4. 
Salatiel García-Nava, Julio C. Ramos-Fernández, Armando I. Martínez-Pérez, et al.

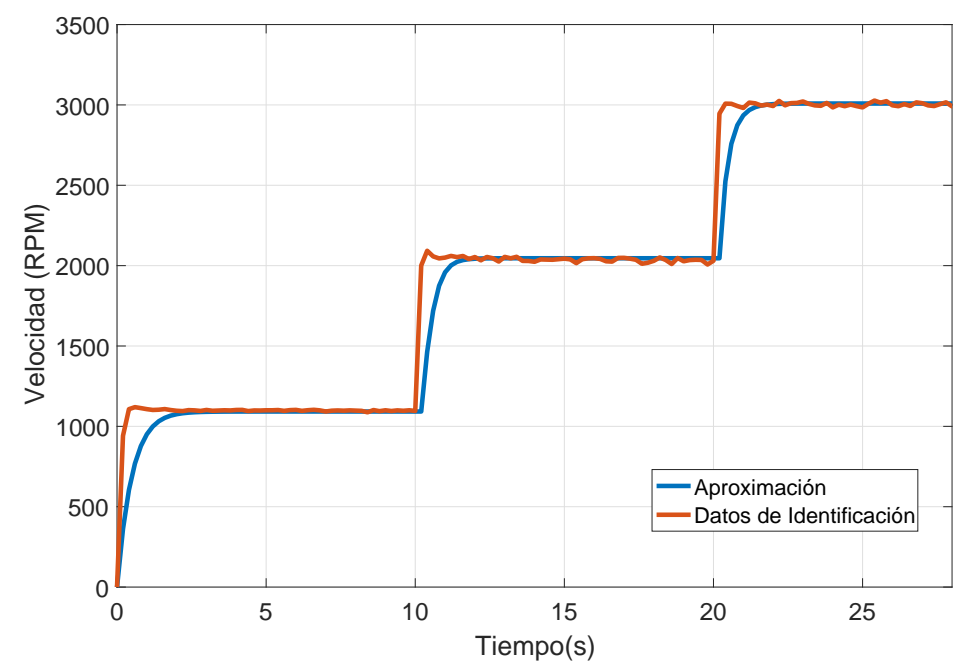

Fig. 3. Respuesta de sub-modelos y datos identificados.

La sintonización de ganancias se llevo a cabo mediante la herramienta tune disponible en el bloque PID de simulik en MATLAB, las ganancias obtenidas de cada sub-modelo identificado se muestran en la Tabla 1.

Tabla 1. La tabla muestra los valores pertenecientes a cada ganancia.

\begin{tabular}{|l|l|l|l|}
\hline Ganancia p & Valor & Ganancia i & Valor \\
\hline$K_{p 1}$ & 0.001829 & $K_{i 1}$ & 0.004233 \\
$K_{p 2}$ & 0.004358 & $K_{i 2}$ & 0.014372 \\
$K_{p 3}$ & 0.003691 & $K_{i 3}$ & 0.010491 \\
\hline
\end{tabular}

4.- Debido a que se realizaron 3 experimentos en el proceso de identificación, se proponen 3 campanas gaussianas, con centros correspondientes a las velocidades en que se hizo estacionaria la respuesta; 1100RPM, 2050RPM y 3000RPM, en este caso $\sigma$ se selecciona de manera equidistante para cubrir el espacio completo de trabajo, las campanas propuestas son mostradas en la Figura 5 .

En donde el valor de disparo $\beta$ en cada sub-modelo es representado por las siguientes ecuaciones $(5,6,7)$ :

$$
\beta_{1}=e^{-\frac{\left(v e l-c_{1}\right)^{2}}{2\left(\sigma_{1}\right)^{2}}}
$$




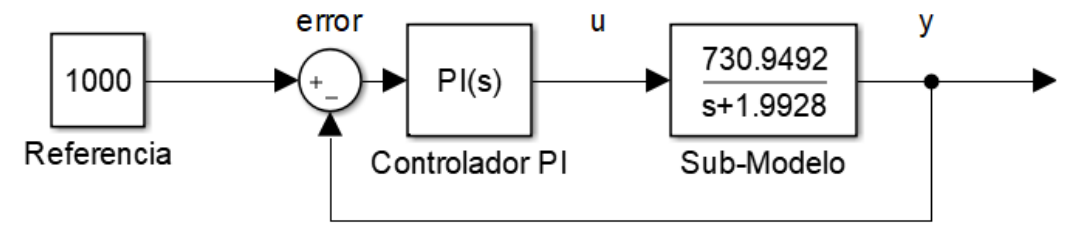

Fig. 4. Diagrama a bloques del controlador clásico aplicado a un submodelo.

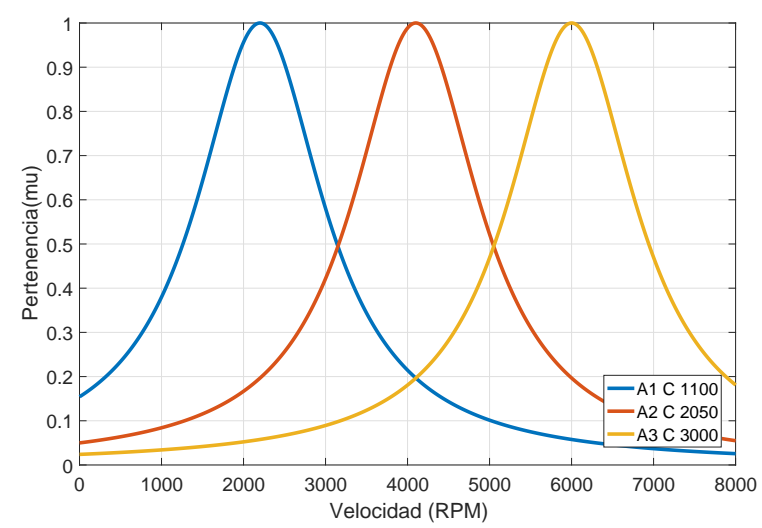

Fig. 5. Campanas Gaussianas propuestas.

$$
\begin{aligned}
& \beta_{2}=e^{-\frac{\left(v e l-c_{2}\right)^{2}}{2\left(\sigma_{2}\right)^{2}}}, \\
& \beta_{3}=e^{-\frac{\left(v e l-c_{3}\right)^{2}}{2\left(\sigma_{3}\right)^{2}} .}
\end{aligned}
$$

Las reglas difusas del controlador se muestra a continuación en las ecuaciones $(8,9,10)$ :

$R_{1}:$ Si $\operatorname{vel}(k)$ está en $A_{1}$ entonces:

$$
u_{1}=\left(\left(K_{p 1}+K_{i 1}\right) e(k)-K_{p 1} e(k-1)+U_{G C}(k-1)\right) \beta_{1},
$$


$R_{2}:$ Si $\operatorname{vel}(k)$ está en $A_{2}$ entonces:

$$
u_{2}=\left(\left(K_{p 2}+K_{i 2}\right) e(k)-K_{p 2} e(k-1)+U_{G C}(k-1)\right) \beta_{2},
$$

$R_{3}:$ Si $\operatorname{vel}(k)$ está en $A_{3}$ entonces:

$$
u_{3}=\left(\left(K_{p 3}+K_{i 3}\right) e(k)-K_{p 3} e(k-1)+U_{G C}(k-1)\right) \beta_{3} .
$$

Se generó una $U_{G C}$ global de control representada por la ecuación (11).

$$
U_{G C}=\frac{\sum_{i=1}^{R}\left(\left(K_{p i}+K_{i i}\right) e(k)-K_{p i} e(k-1)+U_{G C}(k-1)\right) \beta_{i}}{\sum_{i=1}^{R} \beta_{i}},
$$

en donde:

$U_{G C}$ es la señal global de control,

$\beta_{i}$ es el valor de disparo de la i-ésima regla,

$K_{p i}$ es la i-ésima ganancia proporcional del i-ésimo controlador,

$K_{i i}$ es la i-ésima ganancia integrativa del i-ésimo controlador,

$e(k)$ es el error de la velocidad en el k-ésimo evento ( $e=$ referencia velocidad medida),

$e(k-1)$ es el error de la velocidad en un evento anterior $k$.

5.- Finalmente se cierra el lazo de control en la simulación para comprobar el controlador PI difuso propuesto, la respuesta del controlador ante las referencias pedidas es suave como se observa en la Figura 6 .

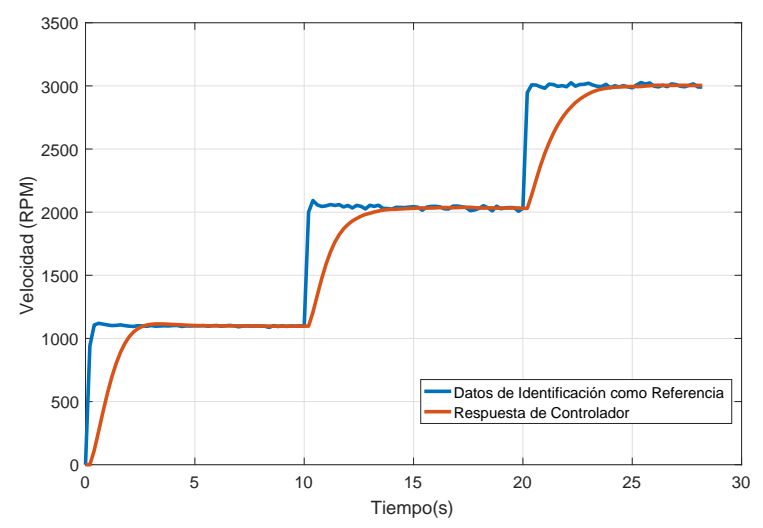

Fig. 6. Respuesta de simulacion PI difuso con ganancias programables. 


\section{Resultados}

Se implementó el controlador en tiempo real al conjunto MIT-VF. En la Figura 7 se muestra el resultado de la regulación en lazo cerrado para tres diferentes referencias de velocidad (1100 RPM, 2050RPM y 3000RPM) en tiempo real.

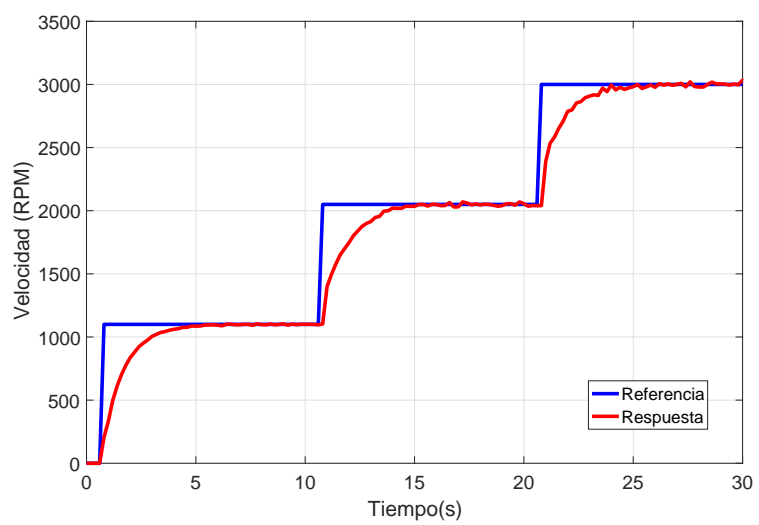

Fig. 7. Respuesta de controlador en tiempo real.

En la Figura 8 se muestra el error de control en lazo cerrado, se observa que el error se aproxima a cero de forma suave.

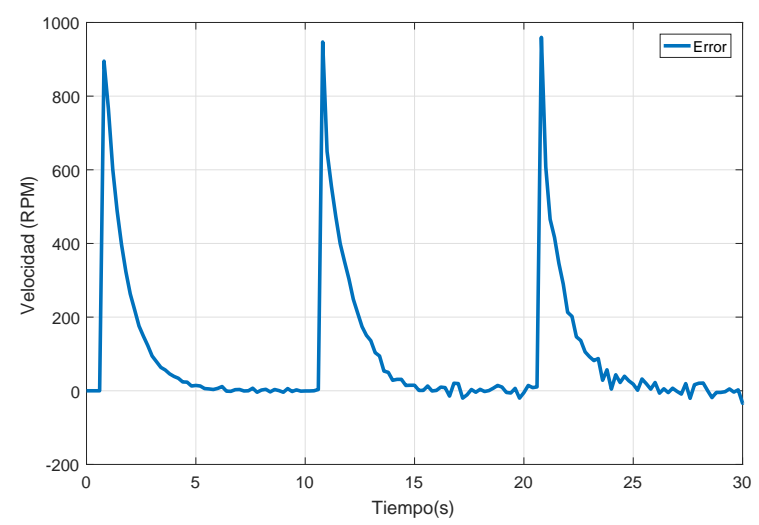

Fig. 8. Error de controlador en tiempo real. 
Salatiel García-Nava, Julio C. Ramos-Fernández, Armando I. Martínez-Pérez, et al.

La ley de control difusa entrega una respuesta de voltaje como se aprecia en la Figura 9, siendo proporcional al error, y suave en todo momento sin presencia de cambios abruptos.

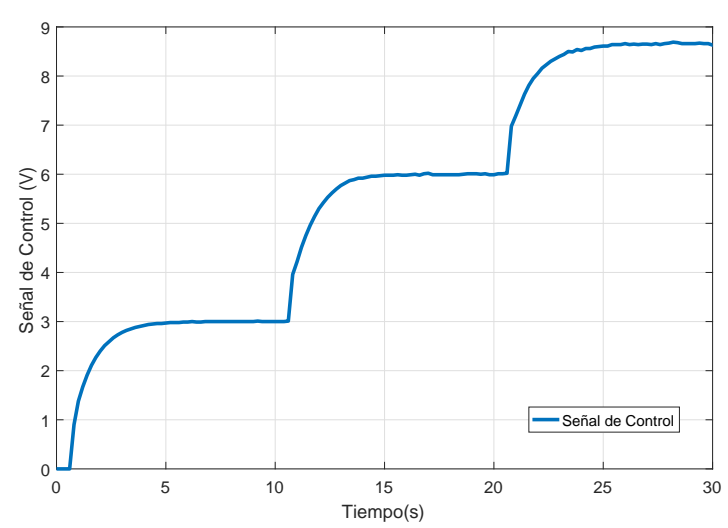

Fig. 9. Señal de Controlador en tiempo real.

Por último se muestra en la Figura 10 un comparativo entre las respuesta de la ley de control en simulación y la respuesta real del sistema ante referencias pedidas.

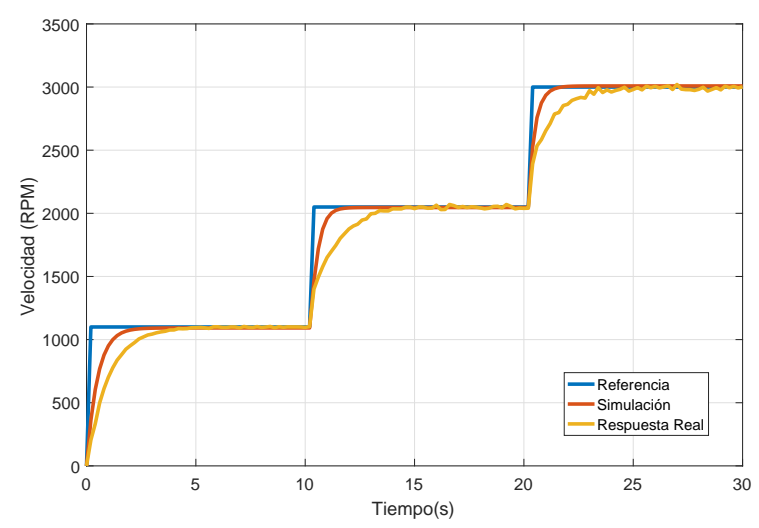

Fig. 10. Respuestas de Controlador en Simulación vs Tiempo Real.

Con los datos obtenidos de la implementación del controlador en tiempo real, se calculó el valor RMSE (Error Cuadrático Medio) mediante la ecuación (12), 
se obtuvieron resultados para cada escalón de velocidad de 4,3, 10,76 y 13,09 respectivamente.

$$
[H] R M S E=\sqrt{\frac{\sum_{i=1}^{n}\left(P_{i}-O_{i}\right)^{2}}{n}}
$$

en donde:

$P_{i}$ es el valor esperado,

$O_{i}$ es la respuesta observada,

$P_{i}-O_{i}$ es el error (Referencia - velocidadmedida), $n$ es el numero de datos utilizados.

Adicionalmente se hace una comparación entre un controlador PI clásico y el PI difuso propuesto aplicado al conjunto MIT-VF en tiempo real, observada en la Figura 11.

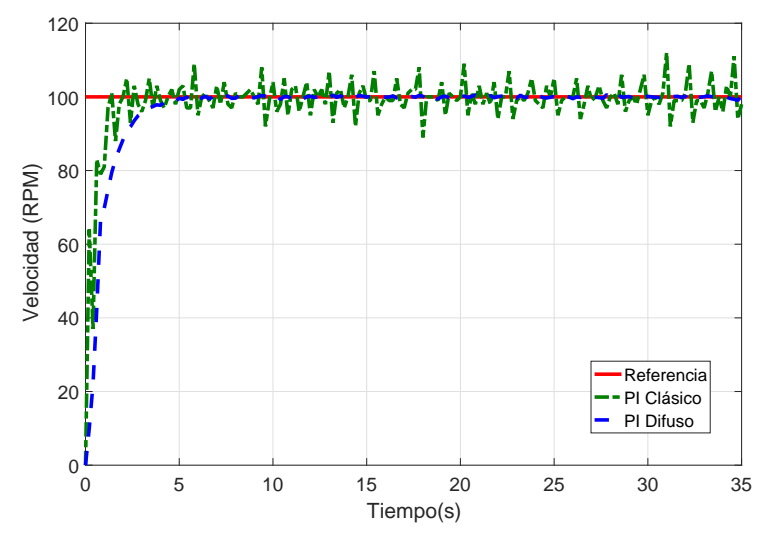

Fig. 11. Respuestas de Controlador Clásico y PI difuso ante una referencia de 100 RPM.

Cabe mencionar que el controlador clásico utilizado como comparativo, visto la Figura 11 mencionada anteriormente, corresponde a un submodelo identificado haciendo uso de la misma metodología, pero correspondiente a una sola región de la dinámica de interés, por lo que al movernos de dicha región presenta oscilaciones en la respuesta, por lo cual se opta por la utilización de un controlador PI difuso de ganancias adaptables.

\section{Conclusiones y trabajos futuros}

En el presente trabajo se muestra una metodología para diseñar leyes de control a sistemas mecatrónicos como es el conjunto VF-MIT, lo cual consiste en la identificación paramétrica de sub-modelos para regiones de interés. A los 
sub-modelos obtenidos se les sintonizaron controles clásicos (en este trabajo controladores PI) implementados en tiempo real, al combinarlos de manera difusa se consigue un controlador más robusto, como se observa en los resultados experimentales. Se concluye que la metodología facilita el trabajo de forma relevante; la identificación y control en sitio del conjunto VF-MIT sin hacer uso de una representación matemáticas no lineal. Todo esto reduce tiempos y costos importantes al llevar a cabo diseño de controladores en aplicaciones industriales y mecatrónicas. En la literatura se mencionan pruebas de estabilidad que se desarrollarán en trabajos futuros.

\section{Referencias}

1. Stephen J. C.: Máquinas Eléctricas. McGraw Hill Higher Education, México (2012)

2. Robert Babubuska: Fuzzy Modeling for Control. Kluwer Academic Publishers, United States of America (1998)

3. Ramos-Velasco, L.E., Ramos-Fernández, J.C., Islas-Gomez, O., et al.: Identificación y control wavenet de un motor de C.A. (RIAI), 2(5), pp. 269-278 (2013)

4. Holtz, J.: Sensorless control of induction motor drives. Proceedings of the IEEE 90(8), pp. 1359-1394 (2002)

5. Finch, J.W., Giaouris, D.: Controlled AC Electrical Drives. Industrial Electronics, IEEE Transactions on. 55, pp. 481-491 (2008)

6. Juhamatti Nikander: Induction Motor Parameter Identification in Elevator Drive Modernization.Thesis. Helsinki University of Technology, Otaniemi (2009)

7. Natsheh, E., Khalid, A.B.: Comparation between Conventional and Fuzzy Logic PID Controllers for Controlling DC Motors. (IJCSI), 7(5), pp. 1694-0814 (2010)

8. Chiewchitboon, P., Tipsuwanpom, V., Soonthomphisaj, N., Piyarat, W.: Speed Control of Three-phase Induction Motor Online Tuning by Genetic Algorithm. Power Electronics and Drive Systems, Power Electronics and Drive Systems (2003)

9. Alolah, A.I.: A New Scheme For Speed Control of Three Phase Induction Motors Using Phase Angle-Controlled Single Phase Supply. Electrical Machines and Drives (2002)

10. El-Barbary, Z.M.S.: Single-to-three phase induction motor sensorless drive system. Alexandria Engineering Journal, 51, pp. 77-83, Elselvier (2012)

11. Mohamed, M.M., Negm-Jamil, M., Bakhashwain, M., Shwehdi, H.: Speed Control of a Three-Phase Induction Motor Based on Robust Optimal Preview Control Theory. IEEE Transactions On Energy Conversion 21(1) (2006)

12. Hasib, A., Amin, H., Ping, H.W., Arop H., Mowed, H.A.F.: Fuzzy Logic Control of a Three Phase Induction Motor Using Field Oriented Control Method. (SICE) (2002)

13. El-Barbary, Z.M.S.: Fuzzy logic based controller for five-phase induction motor drive system. Alexandria Engineering Journal, 51, pp. 263-268 (2012) 